ORIGINAL ARTICLE

\title{
KIT expression in fetal, normal adult, and neoplastic renal tissues
}

\author{
D Miliaras, F Karasavvidou, A Papanikolaou, D Sioutopoulou
}

J Clin Pathol 2004;57:463-466. doi: 10.1136/jcp.2003.013532

See end of article for authors' affiliations .....................

Correspondence to: Dr D Miliaras, Laboratory of Histology and Embryology, Medical School, Aristotle University of Thessaloniki, GR54006 Thessaloniki, Greece; miliaras@auth.gr

Accepted for publication 9 December 2003
Background: KIT is a transmembrane tyrosine kinase receptor, expressed in high amounts in various normal cells. In addition, c-kit mutation or activation is a major pathogenetic event in certain tumours (such as gastrointestinal stromal tumours). There are only limited data in the literature on the expression of KIT in normal and neoplastic renal tissues.

Aims: To investigate KIT expression in normal and neoplastic renal tissues.

Methods: KIT expression was evaluated by means of immunohistochemistry in paraffin wax embedded sections from 67 tissue samples.

Results: Eight of eight fetal kidneys, and 10 of 10 normal adult kidneys revealed cytoplasmic staining of renal tubules. The three cases of renal dysplasia studied expressed KIT in their normal and aberrant tubules. Two of 13 conventional renal cell carcinomas (RCCs), two of seven papillary type RCCs, four of seven chromophobe type RCCs, none of six nephroblastomas, seven of seven oncocytomas, two of two mesoblastic nephromas, and two of four angiomyolipomas were positive.

Conclusion: KIT is expressed in normal fetal and adult renal tubules, and in a subset of renal tumours. The expression of KIT in these renal tumours may prove to have diagnostic relevance and/or therapeutic implications.
$\mathrm{T}$ he growth factor receptor protein KIT (CD117) is encoded by the protooncogene c-kit. The extracellular portion of KIT binds a ligand known as stem cell factor (SCF) and the intracellular domain contains the enzymatic kinase domain. ${ }^{12}$ Transmembrane type III tyrosine kinase, KIT, is phosphorylated as a result of binding with SCF, beginning a cascade of intracytoplasmic signals that are important to cell regulation and development. ${ }^{3}{ }^{4}$ KIT is structurally and functionally related to platelet derived growth factor receptor and macrophage colony simulating factor receptor. Several authors have shown that KIT is constitutionally expressed in various normal tissues: haemopoietic stem cells, mast cells, basal cells of the skin, melanocytes, epithelial cells of the breast, germ cells, interstitial cells of Cajal, and certain regions of the central nervous system..$^{5-10}$ KIT is not expressed in normal squamous epithelium or the glandular epithelium of the endocervix, prostate, stomach, intestine, and pancreas. ${ }^{8}$ In addition, many tumours stain positively with the anti-CD117 antibody, including gastrointestinal stromal tumours (GISTs), mast cell tumours, seminomas, melanomas, endometrial carcinomas, ovarian carcinomas, small cell carcinomas of the lung, and occasionally acute myelogenous leukaemia and ductal breast carcinomas. ${ }^{67910}$ The staining pattern is typically membranous in mast cells and malignant germ cells, and cytoplasmic with membrane accentuation in GISTs. In all other tumours tested, KIT immunoreactivity is cytoplasmic with weak to moderate intensity.

\section{"Many tumours stain positively with the anti-CD117 antibody"}

There are only three reports in the literature regarding KIT expression in normal and neoplastic renal tissues, and only a very small number of cases and very few entities were analysed. ${ }^{611} 12$ Our study was undertaken to examine KIT expression in a wide variety of normal and pathological kidney conditions.

\section{MATERIALS AND METHODS}

Cases were randomly selected from the archives of the departments of pathology of the General Clinic of Thessaloniki and Hippokrateion General Hospital of Thessaloniki, and comprised the following: eight fetal kidneys (16-28 weeks of gestation), 10 normal adult renal tissues, 13 clear cell renal cell carcinomas (RCCs), seven papillary type RCCs, seven chromophobe type RCCs, six nephroblastomas, seven oncocytomas, two mesoblastic nephromas, four angiomyolipomas, and three renal dysplasias (total 67 tissue samples). No patient had received previous treatment. For each case, haematoxylin and eosin stained slides were evaluated to confirm the original diagnosis. Histological typing was according to the guidelines of the Heidelberg and UICC/AJCC classifications of renal tumours. ${ }^{13} 14$ The Fuhrman histological grade was recorded for each case of RCC.

Immunohistochemical detection of CD117 was performed on formalin fixed, paraffin wax embedded tissue sections, $3 \mu \mathrm{m}$ in thickness. High temperature antigen unmasking in ImM EDTA ( $\mathrm{pH} 8$ ) buffer preceded the main procedure: two cycles of 10 minutes in a microwave oven at $500 \mathrm{~W}$. A standardised automated (Nexes; Ventana, Tuscon, Arizona, USA), streptavidin-biotin method (I-VIEW Paraffin DAB; Ventana) followed the application of a monoclonal antibody directed against KIT (clone T595; Novocastra, Newcastle, UK) at a dilution of $1 / 40$. A GIST with diffuse $3+$ positivity for CDl17 was used as positive control. A negative control for immunostaining was carried out by replacing the primary antibody with non-immune rabbit serum. The evaluation system for immunostaining proposed

Abbreviations: CMN, congenital mesoblastic nephroma; GIST, gastrointestinal stromal tumour; RCC, renal cell carcinoma; SCF, stem cell factor 
by Reiner et al was followed..$^{15}$ The intensity of staining was graded as either absent, weak, moderate, or strong ( 0 to 3 scale). The extent of staining was evaluated semiquantitatively and categorised as $0 \%$, less than $10 \%$, between $10 \%$ and $50 \%$, between $50 \%$ and $80 \%$, and more than $80 \%$ (0 to 4 scale). Aggregate scores were obtained for each case (range, $0-7)$; scores of $\geqslant 3$ were regarded as significant (positive cases).

\section{RESULTS}

Immunoreactivity was cytoplasmic in all positive cases. All fetal tissues revealed moderate positivity of proximal tubules (score 5; fig 1A), and weak, focal positivity of distal tubules (score 2). Renal corpuscles were consistently negative. In medullary areas, the loops of Henle showed moderate staining (score 5), whereas collecting tubules were negative. Normal adult renal tissues also showed moderate positivity of proximal and distal tubules (score 5; fig 1B). Renal corpuscles, loops of Henle, and collecting tubules were negative. In the three cases of renal dysplasia studied both normal and dysplastic tubules were positive (score 5), except for widely dilated tubules with flattened attenuated epithelium, which were negative.

Table 1 shows the characteristics of the renal tumours studied. Two of 13 conventional RCCs (scores 3 and 5; fig 1C), two of seven papillary type RCCs (scores 3 and 4), and four of seven chromophobe type RCCs were CDl17 positive (scores 3-5). Only two of 14 low grade RCCs were positive, whereas six of 13 high grade RCCs were positive $(\mathrm{p}=0.07$; Fisher's exact test). Fuhrman's grades I and II were categorised as low grade tumours and Fuhrman's grades III and IV as high grade tumours. All oncocytoma cases revealed diffuse, moderate, or intense immunostaining (scores 5-7; fig 1D), whereas all six nephroblastomas were negative. Both mesoblastic nephromas showed diffuse, moderate to intense positivity of the tumour spindle cells (score 5; fig 1E). Two of four angiomyolipomas contained a few scattered cells that were moderately positive (score 3; fig $\mathrm{lF})$.

\section{DISCUSSION}

RCC is a phenotypically and genetically heterogeneous disease. International agreement has been reached on the histological classification of renal epithelial neoplasms. ${ }^{13}{ }^{14}$ This new classification recognises RCC as comprising morphologically and genetically distinct entities, and it has been noted that the histological classification of renal tumours should be in line with current genetic knowledge. ${ }^{16}$ Recent reports have demonstrated significant differences in patient outcome according to histological subtype using the new UICC/AJCC and Heidelberg classification. ${ }^{17}{ }^{18}$ Although clinical, radiographical, and pathological assessment are considered important prognostic factors for RCC, newer studies are focusing on other laboratory parameters, particularly in the area of biomolecular markers, in an attempt to find factors that will enhance the prognostic evaluation. ${ }^{19}$

KIT belongs to the large family of transmembrane receptors with tyrosine kinase activity, and is important for certain areas of tissue development and maintenance. Commercially available anti-CD117 antibodies have been used to test KIT expression in various normal and neoplastic tissues. ${ }^{5-10}$ However, there is very little information regarding KIT expression in normal and neoplastic renal tissues in the literature. ${ }^{11}{ }^{12}$ Our study shows for the first time that KIT is expressed in the renal tubules of the embryo at least from the 16th week of gestation (and perhaps even earlier). This finding points to a role for KIT in normal kidney development. We also found KIT expression in normal adult renal tubules, an observation that is in agreement with previous studies. $^{6}{ }^{11} 12$

Yamazaki et al are the only authors to have published a study focusing on KIT expression in RCCs. ${ }^{11}$ In their report, which comprised 10 conventional RCCs, two papillary RCCs, and three chromophobe RCCs, only the chromophobe RCCs were found to overexpress KIT by means of immunohistochemistry and reverse transcription polymerase chain reaction. Sequencing analysis of the KIT gene in these three chromophobe RCCs demonstrated no mutation in the region. We also found that KIT was frequently expressed in chromophobe RCCs, but we also found KIT expression in a
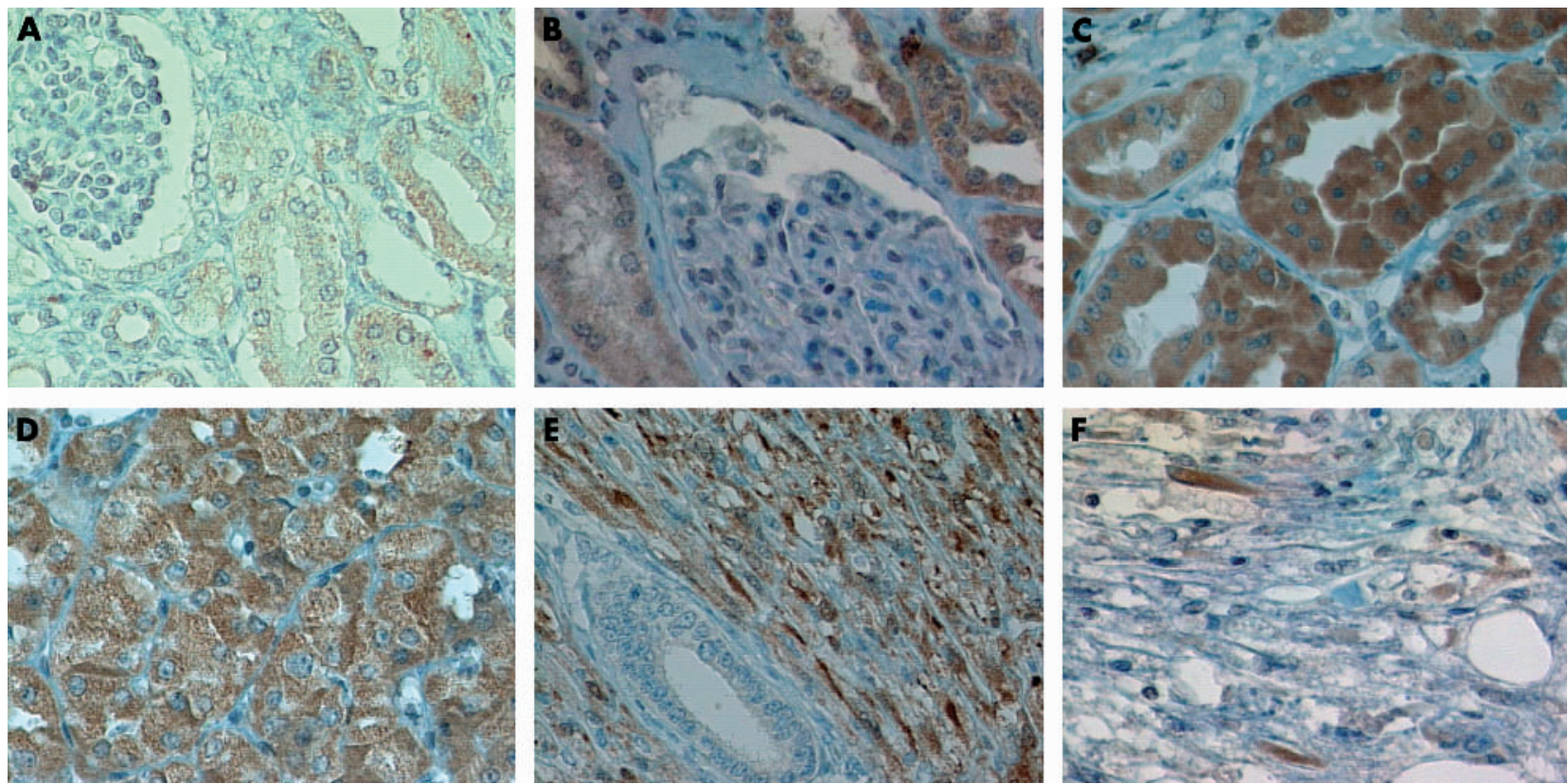

Figure 1 Immunohistochemical expression of KIT. (A) Fetal and (B) adult renal tubules stained for KIT. Note the lack of staining in renal corpuscles. Diffuse intense staining for KIT in (C) conventional renal cell carcinoma, (D) oncocytoma, and (E) mesoblastic nephroma. (F) A few scattered spindle cells were positive for $\mathrm{KIT}$ in angiomyolipoma. 


\begin{tabular}{|c|c|c|c|c|}
\hline Age & Grade & Intensity & Extent & Score \\
\hline \multicolumn{5}{|c|}{ Conventional RCCs } \\
\hline 61 & III & 0 & & \\
\hline 58 & IV & 0 & & \\
\hline 47 & ॥ & 0 & & \\
\hline 66 & III & 0 & & \\
\hline 38 & ॥I & 0 & & \\
\hline 45 & ॥ & 0 & & \\
\hline 77 & ॥I & 0 & & \\
\hline 80 & ॥ & 0 & & \\
\hline 73 & III & 0 & & \\
\hline 70 & IV & 0 & & \\
\hline 72 & III & 3 & 2 & 5 \\
\hline 75 & III & 0 & & \\
\hline 69 & IV & 2 & 1 & 3 \\
\hline \multicolumn{5}{|c|}{ Papillary RCCs } \\
\hline $61 \%$ & ॥ & 0 & & \\
\hline 60 & III & 0 & & \\
\hline 63 & $\|$ & 0 & & \\
\hline 64 & ॥ & 0 & & \\
\hline 69 & III & 1 & 2 & 3 \\
\hline 55 & III & 2 & 2 & 4 \\
\hline 45 & ॥ & 0 & & \\
\hline \multicolumn{5}{|c|}{ Chromophobe RCCs } \\
\hline 51 & III & 2 & 2 & 4 \\
\hline 55 & ॥ & 0 & & \\
\hline 53 & ॥ & 0 & & \\
\hline 59 & ॥ & 1 & 4 & 5 \\
\hline 60 & $\|$ & 1 & 2 & 3 \\
\hline 48 & $\|$ & 0 & & \\
\hline 87 & III & 1 & 4 & 5 \\
\hline \multicolumn{5}{|c|}{ Nephroblastomas } \\
\hline 7 & & 0 & & \\
\hline 10 & & 0 & & \\
\hline 5 & & 0 & & \\
\hline 5 & & 0 & & \\
\hline 6 & & 0 & & \\
\hline 5 & & 0 & & \\
\hline \multicolumn{5}{|c|}{ Oncocytomas } \\
\hline 57 & & 3 & 4 & 7 \\
\hline 73 & & 2 & 4 & 6 \\
\hline 43 & & 1 & 4 & 5 \\
\hline 77 & & 1 & 3 & 4 \\
\hline 64 & & 3 & 4 & 7 \\
\hline 68 & & 2 & 3 & 5 \\
\hline 61 & & 3 & 4 & 7 \\
\hline \multicolumn{5}{|c|}{ Mesoblastic nephromas } \\
\hline 5 days & & 2 & 3 & 5 \\
\hline 3 months & & 3 & 2 & 5 \\
\hline \multicolumn{5}{|c|}{ Angiomyolipomas } \\
\hline 50 & & 0 & & \\
\hline 46 & & 0 & & \\
\hline 68 & & 2 & 1 & 3 \\
\hline 32 & & 2 & 1 & 3 \\
\hline
\end{tabular}

small subset of conventional and papillary type RCCs. There was also a tendency for more frequent KIT expression in higher grade RCCs in our samples, although this association is difficult to establish because different histological types of RCC had different frequencies of KIT expression. In addition, the small numbers of patients studied to date do not allow definite conclusions to be drawn. However, both our study and that of Yamazaki et al suggest that certain RCCs overexpress KIT, and this overexpession may be of clinical importance.

\section{"Our study shows for the first time that KIT is expressed in the renal tubules of the embryo at least from the 16 th week of gestation (and perhaps even earlier)"}

Oncocytomas are considered benign renal neoplasms. Genetic alterations present in oncocytomas are not well defined. ${ }^{16}$ Our study revealed that oncocytomas consistently express KIT, like most of the chromophobe RCCs. In this respect, it is interesting that both oncocytomas and chromophobe RCCs are thought to originate from the intercalated cells of renal collecting tubules, ${ }^{20}{ }^{21}$ whereas clear cell RCCs (which were mostly CD117 negative) probably arise from proximal tubular epithelium. ${ }^{22}$ In addition, the intercalated cells of renal collecting tubules are normally CD117 negative, whereas proximal renal tubules are normally CD117 positive. These findings imply that a mechanism of c-kit activation may be involved in oncocytoma and chromophobe RCC tumorigenesis, and conversely c-kit inactivation may be implicated in conventional RCC carcinogenesis.

Angiomyolipoma is the most common mesenchymal tumour of the kidney. It is thought to originate from perivascular epithelioid cells. ${ }^{23}$ The group of tumours arising from this cell lineage shows a unique pattern of immunoreactivity, with coexpression of melanocyte differentiation markers, such HMB-45 and melan A, in addition to smooth muscle actin. Recently Makhlouf et al studied 15 hepatic and six renal angiomyolipomas and found KIT immunostaining in all of their cases. ${ }^{12}$ Most of them showed diffuse cytoplasmic staining of epithelioid, spindle, round, and fat cells. These authors did not report differences regarding staining intensity between renal and hepatic tumours. They concluded that KIT might be a useful ancillary marker for the diagnosis of angiomyolipoma. However, only two of the four angiomyolipomas revealed moderate cytoplasmic reactivity in a few scattered epitheliod cells in our study. This discrepancy between the two studies may be the result of the different antibodies used. Further studies are needed to clarify whether KIT is consistently expressed in angiomyolipomas.

Congenital mesoblastic nephromas are uncommon renal spindle cell tumours diagnosed in newborns and very young infants, generally within the first 3 months of life. These tumours are characterised by a variably cellular proliferation of bland spindle cells arranged in interlacing bundles, and their clinical behaviour is generally benign. Recently, is has been shown that CMN shares not only its spindle cell morphology with congenital fibrosarcoma, but also a common cytogenetic abnormality, namely: $\mathrm{t}(12 ; 15)(\mathrm{pl} 3 ; \mathrm{q} 26) .^{24}$ This chromosomal translocation results in the ETV6-NTRK3 fusion gene, and is considered a major tumorigenic event for these tumours. Another consistent chromosomal abnormality for these tumours is trisomy 11. However, no involvement of KIT in CMN cytogenetic or molecular genetic studies has been reported to date. Our results suggest that KIT may also be related to CMN biology.

KIT is best studied in GISTs, where constitutive activation of the KIT receptor is a central pathogenetic event. Oncogenic mutations enable the KIT receptor to phoshporylate various substrate proteins, leading to activation of signal transduction cascades that regulate cell proliferation, apoptosis, chemotaxis, and adhesion. ${ }^{25}$ Research into c-kit in GISTs has led not only to a better understanding of the biology of these tumours, but also to therapeutic advances. A newly developed small molecule (STI-571) selectively and competitively binds KIT, resulting in inhibition of tyrosine kinase phosphorylation. This shuts down the transcriptional activity of the cell, resulting in growth arrest. ${ }^{26}$ Targeted treatment to inhibit the kinase activity of KIT is a rational approach to the treatment of KIT positive malignancies. ${ }^{27} 28$ Two key factors are the potency of a given inhibitor and the relative contribution of KIT activation to the growth of the tumour. Given our current understanding of KIT activity in human malignancy, the best candidate diseases for treatment with KIT inhibitors are GISTs, mastocytosis, seminomas, and possibly some cases of acute myelogenous leukaemia. ${ }^{28}$ In addition, KIT inhibitors may play an adjunctive role in diseases such as small cell lung cancer, in which KIT 


\section{Take home messages}

- KIT was expressed in normal fetal and adult renal tubules, and in a subset of renal tumours - clear cell, papillary type, and chromophobe cell renal cell carcinomas, all oncocytomas, and mesoblastic nephromas

- The expression of c-kit in these renal fumours may prove to have diagnostic relevance and/or therapeutic implications

activation is secondary to ligand binding rather than an acquired mutation. It is plausible to speculate that KIT positive RCCs are also candidates for such targeted treatments.

We conclude that KIT is invariably expressed in normal fetal and adult renal tubules, presumably playing an essential role in cell regulation and maintenance. Even though most of the common renal tumours are CD117 negative (conventional RCCs and nephroblastomas), a subset of clear cell, papillary type, and chromophobe cell RCCs, all oncocytomas, and mesoblastic nephromas are immunopositive. CD117 expression may aid histological diagnosis in some of these tumours (for example, oncocytomas). Further studies are needed to clarify whether KIT expression in renal tumours results from c-kit gene mutations or represents an epigenetic phenomenon. In addition, patients with malignant renal tumours expressing KIT may benefit from treatments targeted against this molecule.

\section{Authors' affiliations}

D Miliaras, Laboratory of Histology and Embryology, Medical School, Aristotle University of Thessaloniki, GR54006 Thessaloniki, Greece

F Karasavvidou, Department of Pathology, General Clinic of Thessaloniki, GR54645 Thessaloniki, Greece

A Papanikolaou, D Sioutopoulou, Department of Pathology, Hippokrateion General Hospital, GR54639 Thessaloniki, Greece

\section{REFERENCES}

1 Majumber S, Brown K, Qiu FH, et al. c-kit protein, transmembrane kinase: identification in tissues and characterization. Mol Cell Biol 1988;8:4896-903.

2 Blume-Jensen P, Claesson-Welsh L, Siegbahn A, et al. Activation of the human c-kit protein by ligand-induced dimerization mediates circular actin reorganization and chemotaxis. EMBO J 1991;10:4121-8.

3 Ashman LK. The biology of stem cell factor and its receptor c-kit. Int J Biochem Cell Biol 1999;31:1037-51.
4 Taylor ML, Metcalfe DD. KIT signal transduction. Hematol Oncol Clin North Am 2000; 14:517-35

5 Gibson PC, Cooper K. CD1 17 (KIT): a diverse protein with selective applications in surgical pathology. Adv Anat Pathol 2002;9:65-9.

6 Natali PG, Nicotra MR, Sures I, et al. Expression of c-kit receptor in normal and transformed human non-lymphoid tissues. Cancer Res 1992;52:6139-43

7 Arber DA, Tamayo R, Weiss LM. Paraffin section detection of the c-kit gene product (CD117) in human tissues: value in the diagnosis of mast cell disorders. Hum Pathol 1998;29:498-504.

8 Lammie A, Drobnjak M, Gerald W, et al. Expression of c-kit and kit ligand proteins in normal human tissues. J Histochem Cytochem 1994;42:1417-25.

9 Mietinnen M, Lasota J. Gastrointestinal stromal tumors: definition, clinical, histological, immunohistochemical and molecular genetic features and differential diagnosis. Virchows Arch 2001;438:1-12.

10 Tsuura Y, Hiraki H, Watanabe K, et al. Preferential localization of c-kit product in tissue mast cells, basal cells of the skin, epithelial cells of breast, small cell lung carcinoma, and seminoma/dysgerminoma in human: immunohistochemical study of formalin-fixed, paraffin-embedded tissues. Virchows Arch 1994;424:135-41.

11 Yamazaki K, Sakamoto M, Ohta T, et al. Overexpression of KIT in chromophobe renal cell carcinoma. Oncogene 2003;22:847-52.

12 Makhlouf HR, Remotti HE, Ishak KG. Expression of KIT (CD1 17) in angiomyolipoma. Am J Surg Pathol 2002;26:493-7.

13 Kovacs G, Akhtar M, Beckwith BJ, et al. The Heidelberg classification of renal cell tumours. J Pathol 1997; 183:131-3.

14 Störkel S, Eble JN, Adlakha K, et al. Classification of renal cell carcinoma: workgroup no. 1. Cancer 1997;80:987-9.

15 Reiner A, Neumeister B, Spona J, et al. Immunocytochemical localization of estrogen and progesterone receptor and prognosis in human primary breast cancer. Cancer Res 1990;50:7057-61.

16 Moch H, Mihatsch MJ. Genetic progression of renal cell carcinoma. Virchows Arch 2002;441:320-7.

17 Cheville JC, Lohse CM, Zincke $\mathrm{H}$, et al. Comparisons of outcome and prognostic features among histologic subtypes of renal cell carcinoma. Am J Surg Pathol 2003;27:612-24.

18 Liungberg B, Alamdari Fl, Stenling R, et al. Prognostic significance of the Heidelberg classification of renal cell carcinoma. Eur Urol 1999;36:565-9.

19 Srigley JR, Hutter RVP, Gelb AB, et al. Current prognostic factors-renal cell carcinoma. Cancer 1997;80:994-6.

20 Störkel S, Pannen B, Thoenes W, et al. Intercalated cells as a probable source for the development of renal oncocytoma. Virchows Arch B Cell Pathol Mol Pathol 1988;56:185-9.

21 Störkel S, Steart PV, Drenckhahn D, et al. The human chromophobe cell renal carcinoma: its probable relation to intercalated cells of the collecting duct. Virchows Arch B Cell Pathol Mol Pathol 1989;56:237-45.

22 Krishnan B, Truong LD. Renal epithelial neoplasms: the diagnostic implications of electron microscopic study in 55 cases. Hum Pathol 2002;33:68-79.

23 Bonetti F, Pea M, Martignoni G, et al. The perivascular epithelioid cell and related lesions. Adv Anat Pathol 1997:4:343-58.

24 Sandberg AA. Updates on the cytogenetics and molecular genetics of bone and soft tissue tumors congenital (infantile) fibrosarcoma and mesoblastic nephroma. Cancer Genet Cytogenet 2002;132:1-13.

25 Heinrich M, Rubin BP, Longley J, et al. Biology and genetic aspects of gastrointestinal stromal tumors: KIT activation and cytogenetic alterations. Hum Pathol 2002:33:484-95.

26 Joensuu $H$, Fletcher $C$, Dimitrijevic $S$, et al. Management of malignant gastrointestinal stromal tumours. Lancet Oncol 2002;3:655-64.

27 Krystal GW, Honsawek S, Litz J, et al. The selective tyrosine kinase inhibitor STI571 inhibits small cell lung cancer growth. Clin Cancer Res 2000;6:3319-26.

28 Heinrich MC, Blanke CD, Druker BJ, et al. Inhibition of KIT tyrosine kinase activity: a novel molecular approach to the treatment of KIT-positive malignancies. J Clin Oncol 2002;20:1692-703. 